\title{
GRACE SIGNAL FILTERING AS A MEANS OF DETERMINING EQUIVALENT WATER THICKNESS IN POLAND
}

\author{
MONIKA BIRYŁO ${ }^{1}$, JOLANTA NASTULA ${ }^{2}$ \\ ${ }^{1}$ University of Warmia and Mazury, Department of Astronomy and Geodynamics \\ Heweliusza St. 10/2, 10-724 Olsztyn, Poland \\ monika.sienkiewicz@uwm.edu.pl \\ 2 Social Academy of Science \\ Sienkiewicza St. 9, 90-113 Łódź, Poland \\ nastula@cbk.waw.pl
}

\begin{abstract}
In the paper an Equivalent Water Thickness (EWT) determination as a way of observing gravity variations is described. Since raw data acquired directly from Gravity Recovery and Climate Experiment - GRACE satellites is unsuitable for analysis due to stripes occurrence, a filtering algorithm must be used. In this paper, authors are testing two isotropic (Gauss, CNES/GRGS) filters and two anisotropic filters (Wiener-Kolomogorov, ANS). Correlation, amplitude ratio, and modification were determined as well as maps were generated.
\end{abstract}

KEY WORDS: GRACE, Equivalent Water Thickness, filter.

\section{INTRODUCTION}

Due to the fact that for the Equivalent Water Thickness (EWT) determination different processing of the raw data, different methods of filtration, and the degree of spherical harmonics expansion are used, the results may significantly differ in the spatial scale. Ignoring these differences can lead to an incorrect interpretation of the 
results. Therefore, it is necessary to estimate the differences and keep check Gravity Recovery and Climate Experiment - GRACE of signal modifications. It may have great importance in the water balance studies in the regional scale, where signal loss can be significant, and appreciably influence the final value of the EWT. In such cases, due to large GRACE signal loss, the water balance cannot be closed, since it causes errors in the residuum (Klees et al. 2007, Swenson 2011). The Equivalent Water Thickness is a way of representing gravity variations (changes are expressed in millimeters of EWT).

\section{EQUIVALENT WATER THICKNESS DETERMINATION}

For gravity determination the best option is spherical harmonic $(\mathrm{SH})$ usage. Relation between the Earth density and gravity potential is presented by the following formula (Liu 2008):

$$
\left\{\begin{array}{l}
\Delta \bar{C}_{l, m} \\
\Delta \bar{S}_{l, m}
\end{array}\right\}=\frac{\delta_{a v}(2 l+1)}{3 \delta_{w}\left(1+k_{l}\right)}\left\{\begin{array}{l}
\Delta C_{l, m} \\
\Delta S_{l, m}
\end{array}\right\}
$$

Where: $\delta_{a v}$ denotes the average Earth density $\left(5517 \mathrm{~kg} / \mathrm{m}^{3}\right), k_{l}$ is the load Love number of 1 degree, and $\delta_{w}$ is the fresh sweet water density.

The gravity potential is expressed with the formula:

$$
V(\varphi, \lambda, r)=\sum_{l=0}^{\infty} r^{-(l+1)} \sum_{m=0}^{l}\left(a_{l, m} \cos m \lambda+b_{l, m} \sin m \lambda\right) P_{l, m}(\sin \varphi)
$$

Where: $\varphi$ is the geographical latitude, $\lambda$ - the geographical longitude, $P_{l, m}(\sin \varphi) \cos m \lambda$ and $P_{l, m}(\sin \varphi) \sin m \lambda$ are ratio of associated Legendre functions of cosine and sine coefficients and are called spherical harmonics, and expressions for $a_{l, m}$ i $b_{l, m}$ are:

$$
\begin{gathered}
a_{l, m}=\sqrt{\frac{(2 l-1)(2 l+1)}{(l-m)(l+m)}} \\
b_{l, m}=\sqrt{\frac{(2 l+1)(l+m-1)(l-m-1)}{(l-m)(l+m)(2 l-3)}}
\end{gathered}
$$

The associated Legendre function of degree $l$ and order $m$ can be expressed as follows:

$$
P_{l, m}(\sin \varphi)=\cos ^{m} \varphi \frac{d^{m} P_{l}(\sin \varphi)}{(d \sin \varphi)^{m}}
$$

where $P_{n, m}(\sin \varphi)$ is the Legendre polynomial of degree $l$ and order $m$. 
Taking into account formula 2 on gravity potential, and adding relation expressed in formula 1 and filtering algorithm, it becomes possible to derive an Equivalent Water Thickness (Chambers 2006):

$$
\delta N(\varphi, \lambda, r)=R \sum_{l=2}^{\infty} \sum_{m=0}^{l}\left(\bar{C}_{l, m} \cos m \lambda+\bar{S}_{l, m} \sin m \lambda\right) \bar{P}_{l, m}^{n}(\sin \varphi) W_{n} \frac{(2 l+1)}{\left(1+k_{n}\right)}
$$

where: $\bar{P}_{l, m}^{n}(\sin \varphi)$ is the normalized Legendre polynomial of degree $n$ and order $m$, $k_{n}$ - the load Love number of degree $n . W_{n}$ is a filter.

\section{GRACE DATA FILTERING}

GRACE data elaboration is executed in three stages:

- pre-processing,

- processing,

- post-processing.

For the purpose of the present paper, already processed data were acquired, as the aim was to test post-processing algorithms. A filtering algorithm is dependant on spatial wavelength (of degree $l$ ) and orientation (of order $m$ ). Filtering is a mathematical process whose purpose is to limit noise in original GRACE data. The easiest way to eliminate bias which occurs in a higher degree and order of spherical harmonics, is cutting of higher terms of the $\mathrm{SH}$ expansion. The disadvantage of such solution is a signal strength reduction (Tapley and Reiger 2008). Another method of the Equivalent Water Thickness determination relies on the use of an averaging filter, whose purpose is a noise reduction through calculation of weighted mean values of neighboring points. Employing a spatial filter allows for elimination of systematic errors that occur especially in higher degree coefficients. Chambers (2006) ascertained that systematic errors appear from $8^{\text {th }}$ degree and order and have different structure for odd and even coefficients.

\section{FILTERS USED FOR GRACE DATA ELABORATION}

When gravity is determined on the basis of GRACE data, a signal can be filtered using:

- isotropic filters, or

- anisotropic filters.

Filters from groups mentioned above have both, advantages and disadvantages. Filtering effectiveness is dependant on parameters such as: filtering radius, GRACE signal recognition, liquid medium model usage, gravity potential expansion.

Gauss isotropic filter is well-known and easy to implement, but it does not reduce any of errors. Applying a Gauss filter results in elimination of stripes in raw figuration 
of GRACE data. A great disadvantage of the filter is signal strength reduction that can reach as much as $28 \%$. Gauss filter is described by the formula (Chambers 2006):

$$
W_{n}=\exp \left[-\frac{\left(n r / a_{E}\right)^{2}}{4 \ln (2)}\right]
$$

where $r$ is the filtering radius which is dependant on type of tested issue or area (Veselinova-Renglova 2007). When the Gauss filter is used, only the degree does the job - only the wavelength has an effect (Chambers 2006).

The second checked isotropic filter was according to the algorithm used by a computing center, CNES/GRGS:

$$
W_{n}=\left[\frac{4 \Pi G r \rho_{W}}{(2 n+1) \gamma(\varphi)}\right]\left(1+k_{n}\right)
$$

where: $k_{n}$ is the Love number, $\gamma(\varphi)$ - the normal acceleration.

But the GRACE signal structure is anisotropic, so the best way is to use a filter which is dependant on degree and order at the same time. In such case, equation 6 is being replaced by (Han et al. 2005):

$$
\delta N(\varphi, \lambda, r)=\frac{1}{4 \Pi} \sum_{l=2}^{\infty} \sum_{m=0}^{l} \bar{P}_{l, m}(\sin \varphi)\left\{G_{l, m}^{C} \cos m \lambda+G_{l, m}^{S} \sin m \lambda\right\}
$$

where:

$$
\begin{aligned}
G_{l, m}^{C} & =W_{l, m} \bar{P}_{l, m} \cos \varphi \cos m \lambda \\
G_{l, m}^{S} & =W_{l, m} \bar{P}_{l, m} \cos \varphi \sin m \lambda
\end{aligned}
$$

Such filter, dependant on degree and order, can be expressed by (Veselinova-Renglova 2007):

$$
W_{l, m}=\frac{\operatorname{var}\left(K_{l, m}, K_{l, m}\right)}{\operatorname{var}\left(K_{l, m}, K_{l, m}\right)+\sigma^{2} K_{l, m}}
$$

where: $K_{l, m}=\left\{C_{l, m}, S_{l, m}\right\}$, and $\sigma^{2} K_{l, m}$ is the variance of $K_{l, m}$ coefficient error.

All anisotropic filters take into account noise variance. The higher is $\mathrm{SH}$ expansion degree and order, the higher is the error (Fig. 1).

The variance of a GRACE signal and error is expressed by (Veselinova-Renglova 2007):

$$
\begin{gathered}
s_{l}^{2}=\sum_{m=0}^{l}\left(\bar{C}_{l, m}^{2}+\bar{S}_{l, m}^{2}\right) \\
\sigma_{l}^{2}=\sum_{m=0}^{l}\left(\sigma_{C_{l, m}}^{2}+\sigma_{S_{l, m}}^{2}\right)
\end{gathered}
$$




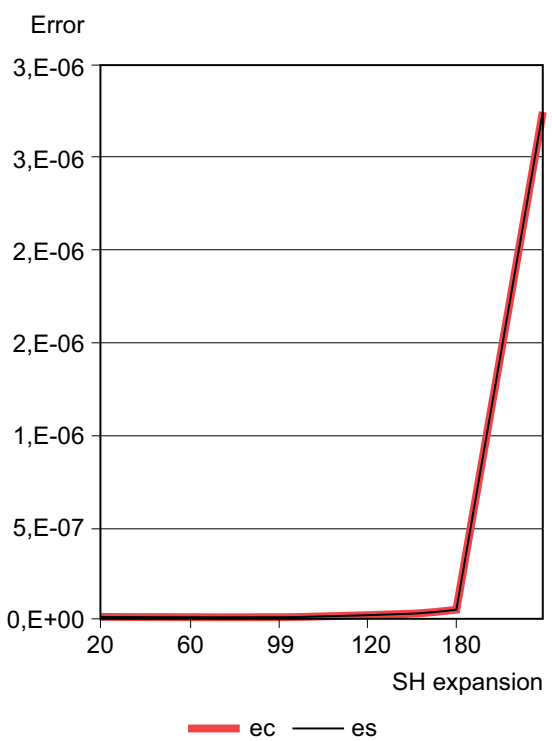

Figure 1. Dependence of error value on spherical harmonic expansion

If equations 13 and 14 are applied to formula (12), the Wiener-Kolomogorov filter formula can obtains (Veselinova-Renglova 2007):

$$
W_{l}=\frac{s_{l}^{2}}{s_{l}^{2}+\sigma_{l}^{2}}
$$

The second anisotropic filter (called Anisotropic Non-Symmetric Filter - ANS) tested in the work described here is a filter used in TU Delft as an alternative to Wiener-Kolomogorov filter. Mass variations are defined by (Zhang 2009):

$$
f_{w}(x)=\frac{1}{4 \Pi R^{2}} \sum_{n=0}^{N}(f(y) W(x, y))
$$

where the $W(x, y)$ filter is as follows (Klees 2008):

$$
W(x, y)=\sum_{l, m} \sum_{p, q} W_{l m, p q} Y_{l, m}^{\prime}(\xi) Y_{p, q}^{\prime}(\eta)
$$

where: $l, m$ are the degree and order, $\mathrm{Y}^{\prime}{ }_{l, m}$ is the normalized $\mathrm{SH}, W_{l m, p q}$ is the filtering coefficient, and $\xi \mathrm{i} \eta$ are coordinates of a point on the sphere. 


\section{TESTS}

\section{CORRELATION BETWEEN UNFILTERED AND FILTERED MODEL}

The first step of testing algorithms used for filtering GRACE signal, was model correlation. Correlation between raw, unfiltered model and filtered model is different for isotropic and anisotropic algorithm. When a filter was used, results were as follow:

- for the Gauss filter with $300 \mathrm{~km}$ filtering radius - correlation of $0.8 \div 1$ till $\mathrm{SH}$ expansion 40, and no correlation from SH expansion 120;

- for the Gauss filter with $500 \mathrm{~km}$ filtering radius - correlation of $0.8 \div 1$ till $\mathrm{SH}$ expansion 35, and no correlation from SH expansion 60;

- for the Gauss filter with $700 \mathrm{~km}$ filtering radius - correlation of $0.8 \div 1$ till $\mathrm{SH}$ expansion 30, and no correlation from $\mathrm{SH}$ expansion 35;

- for the CNES/GRGS filter - correlation of 0.8 for whole C component, and for $\mathrm{S}$ component from the degree and order 3 ;

- for the ANS filter - correlation equal to 1 for the whole SH expansion;

- for the Wiener - Kolomogorowv filter - correlation equal to 0.9 for the whole SH expansion.

\section{AMPLITUDE RATIO DETERMINATION}

Also, an amplitude ratio was tested for determination GRACE signal reduction. As it was shown in Figure 1, the more terms of spherical harmonic expansion is taken for calculation, the higher error is introduced. On the other hand, as many terms as possible of SH expansion terms should be used for assuring high GRACE signal strength. The idea is to optimize both, signal strength and error range, and thus finding the best number of $\mathrm{SH}$ expansion terms that should be used for post processing.

When analyzing low degree of $\mathrm{SH}$ expansion, a reduction up to $28 \%$ of signal strength was found for the Gauss filter. Experimenting with these filters indicated that better results (i.e. less signal strenth reduction) can be achieved while adapting longer filtering radius. For the best signal strength maintenance, usage of $118 \pm 10$ terms is recomended when filtering with the Gauss filter. The CNES/GRGS and Wiener-Kolomogorov filters indicated $6 \%$ reduction of GRACE signal strength, and the ANS filter has shown $0 \%$ loss.

\section{SIGNAL MODIFICATION}

Every interpolated point represents some kind of average of a group of signals. When comparing filtered value of EWT with unfiltered one, a quantity of signal modification can be determined. A signal modification test was based on this formula (Swenson 2011): 


$$
\sum_{i}^{N}\left(S_{i}^{P}-A S_{i}^{F}\right)^{2}
$$

Where: $S_{i}^{P}$ is the unfiltered value of EWT in a month i, $S_{i}^{F}$ - filtered EWT value in a month i, $N$ - the number of months included in the analysis, $A$ - a constant (scalling factor). The best solution is the one which allows A value become 1. This means that $S_{i}^{P}$ takes the same values as does $S_{i}^{F}$.

Table 1. GRACE signal modification

\begin{tabular}{|c|c|c|c|c|c|c|c|}
\hline \multicolumn{2}{|c|}{$\begin{array}{c}\text { Geo- } \\
\text { position }\left[{ }^{\circ}\right]\end{array}$} & $\begin{array}{c}\mathrm{S}(\mathrm{P})- \\
\mathrm{S}\left(\mathrm{F}_{-} \text {CNES/GRGS }\right)\end{array}$ & $\begin{array}{c}\mathrm{S}(\mathrm{P})- \\
\mathrm{S}\left(\mathrm{F} \_ \text {gauss300 }\right)\end{array}$ & $\begin{array}{c}\mathrm{S}(\mathrm{P})- \\
\mathrm{S}\left(\mathrm{F} \_ \text {gauss500) }\right.\end{array}$ & $\begin{array}{c}\mathrm{S}(\mathrm{P})- \\
\mathrm{S}\left(\mathrm{F} \_ \text {gauss700 }\right)\end{array}$ & $\begin{array}{c}\mathrm{S}(\mathrm{P})- \\
\mathrm{S}\left(\mathrm{F}_{-} \text {wiener }\right)\end{array}$ & $\begin{array}{c}\mathrm{S}(\mathrm{P})- \\
\mathrm{S}\left(\mathrm{F}_{-} \text {ANS }\right)\end{array}$ \\
\hline$\varphi$ & $\lambda$ & \multicolumn{6}{|c|}{$[\mathrm{mm}]$} \\
\hline 59 & 9 & 0.03 & 0.00 & 0.00 & 0.00 & 0.37 & 0.31 \\
\hline 59 & 10 & 0.03 & 0.00 & 0.00 & 0.00 & 0.46 & 0.30 \\
\hline 59 & 11 & 0.03 & 0.00 & 0.00 & 0.00 & 0.44 & 0.28 \\
\hline 60 & 9 & 0.02 & 0.00 & 0.00 & 0.00 & 0.03 & 0.25 \\
\hline 60 & 10 & 0.02 & 0.00 & 0.00 & 0.00 & 0.06 & 0.26 \\
\hline 60 & 11 & 0.03 & 0.00 & 0.00 & 0.00 & 0.02 & 0.23 \\
\hline 61 & 9 & 0.02 & 0.00 & 0.00 & 0.00 & 5.12 & 0.21 \\
\hline 61 & 10 & 0.02 & 0.00 & 0.00 & 0.00 & 5.23 & 0.22 \\
\hline 61 & 11 & 0.02 & 0.00 & 0.00 & 0.00 & 6.87 & 0.18 \\
\hline
\end{tabular}

From Table 1 it can be concluded that the greatest signal modification took place when the Wiener-Kolomogorov filter has been used $(0.06 \div 6.87)$ and range of values made it impossible to eliminate an error of modification (there is no possibility to determine a value of the scalling factor A because of discrepancies of modification values). On the other hand, the best result can be seen when the Gauss filter was used, and the higher was SH expansion, the smaller was signal modification (0.00). Moreover, the longer filtering radius applied, the more uniform modification resulted. Although, using the CNES/GRGS filter showed modification rates $0.02 \div 0.03$, but the result is uniform and can be eliminated by using the scaling factor A. When using the ANS filter a modification range $0.18 \div 0.31$ was found, but there still remains a possibility to reduce it with the scaling factor.

\section{MAPS OF EWT IN POLAND}

The last test was based on generating maps (with a step 3 degrees/3 degrees) of unfiltered signal and filtered with described algorithms. Results are presented in Figure 2. 
a)

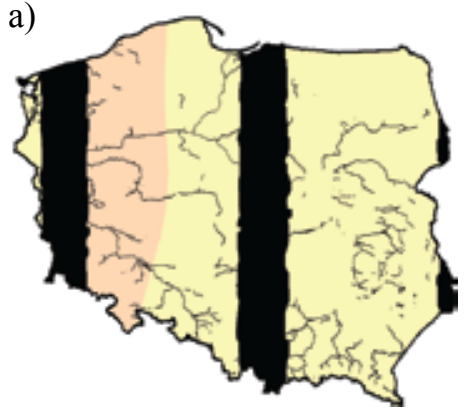

b)

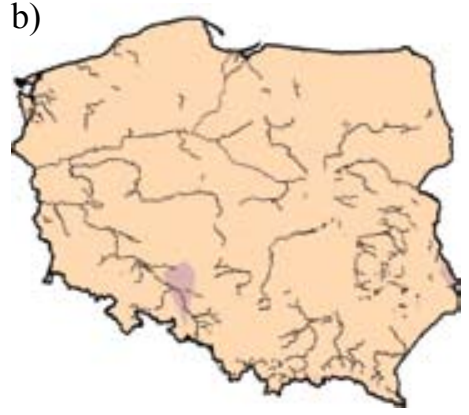

c)

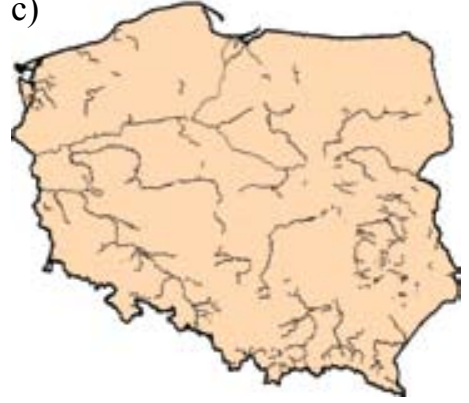

d)

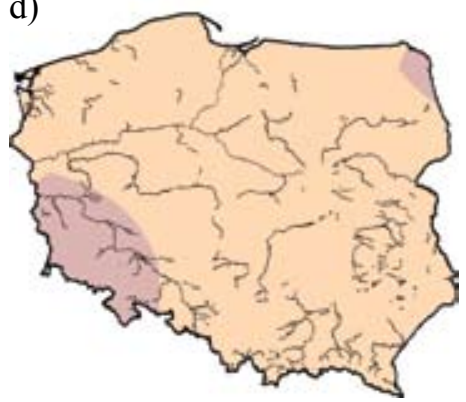

e)

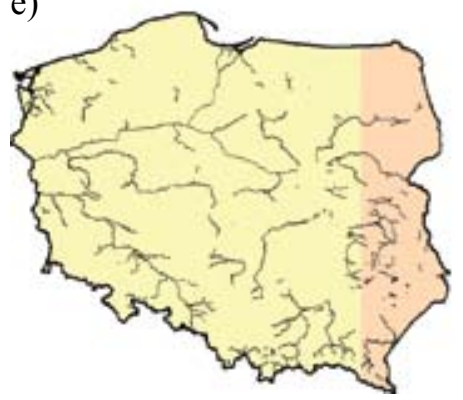

f)

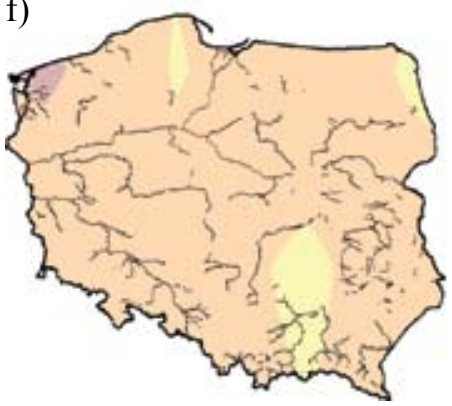

g)

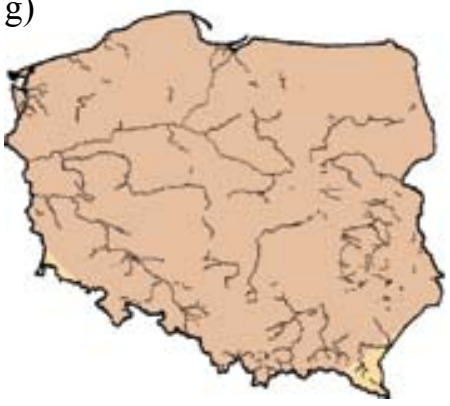

$[\mathrm{cm}]$

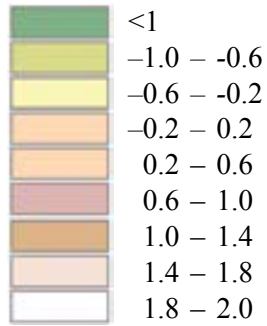

Figure 2. Spatial distribution of the Equivalent Water Thickness calculated without filtering (a), and with the following filters: Gauss filter of: $300-\mathrm{km}$ radius (b), $500-\mathrm{km}$ radius (c), 700-km radius (d), CNES/GRGS filter (e), Wiener- Kolomogorov filter (f), ANS filter (g) (Contours of Poland taken from: http://www.eea.europa.eu/data-and-maps/data/ biogeographical-regions-europe-2008/zipped-shapefile-format-vector-polygon) 
Figure 2 depicts the Equivalent Water Thickness range rate. The first map (Fig. 2a) shows the EWT without filtering. When using the Gauss filter with $300 \mathrm{~km}$ radius (Fig. 2b), $500 \mathrm{~km}$ radius (Fig. 2c), $700 \mathrm{~km}$ radius (Fig. 2d), Wiener-Kolomogorov filter (Fig. 2f), ANS filter (Fig. 2g) more or less the same spatial range obtains $(-0.2 \div 0.2 \mathrm{~cm})$. With the CNES/GRGS filter, vertical spatial distribution was found to have values $-1 \div 1 \mathrm{~cm}$.

\section{CONCLUSIONS}

In this paper analyzis of GRACE data filtering was presented. Authors carried out four tests of filters, which included correlation, amplitude ratio and signal modification and EWT maps generation. On a basis of this research it can be concluded that:

- The best results in the four tests were achieved when using ANS filter;

- Correlation is strongly dependant on the filter used. For the Gauss filter correlation is $0.8 \div 1$ when low number of SH expansion terms are taken for computation $(30-40)$, and gradully decreases to 0 (the longer filtering radius is taken for calculation, the quicker is decrease to 0 ); for the CNES/GRGS filter correlation is on a level of 0.8 , for the ANS filter correlation is equal to 1 , for the Wiener-Kolomogorov filter correlation is equal to 0.9 ;

- Analysis of amplitude ratio has shown that low degree of SH expansion leads to up to $28 \%$ reduction of signal strength for the Gauss filter and better results can be achieved by adapting longer filtering radius and inclusion of $118 \pm 10$ terms of $\mathrm{SH}$ expnsion. The CNES/GRGS and Wiener-Kolomogorov filters indicated $6 \%$ reduction of GRACE signal strength, and the ANS filter performed with $0 \%$ signal loss;

- The greatest signal modification was when the Wiener-Kolomogorov filter has been used $(0.06 \div 6.87)$, and the best results can be seen for the Gauss filter (even $0.00)$ - the longer filtering radius, the more uniform modification. Using the CNES/ GRGS filter led to modification rates of $0.02 \div 0.03$, and using the ANS filter it ranged from 0.18 to 0.31 ;

- The same spatial range $(-0.2 \div 0.2 \mathrm{~cm})$ was found for the Gauss filter, WienerKolomogorov filter, and ANS filter, but for the CNES/GRGS filter, the vertical spatial distribution was within the range $-1 \div 1 \mathrm{~cm}$.

\section{ACKNOWLEDGEMENTS}

This work was supported by the National Science Centre, contract number NN 526157040 and 2145/B/T02/2011/40 of May 16, 2011. 


\section{REFERENCES}

Chambers D.P., 2006, Converting Release-04 Gravity Coefficient into Maps of Equivalent Water Thickness, Geophysical Research Letters.

Han S.-C., Shum C.K., Jekeli C., Kuo C.-Y., Wilson C., Seo K.-W., 2005, Non-isotropic filtering of GRACE temporal gravity for geophysical signal enhancement, Geophysical Journal International, 163, 9.

Klees R., Zapreeva E.A., Winsemius H.V., Savenije H.H.G., 2007, How to properly scale GRACE estimates of water storage variations, Proceedings of the 1st International Symposium of the International Gravity Field Service, "Gravity field of the Earth", Istanbul.

Klees R., Liu X., Wittwer T., Gunter B.C., Revtova E.A., Tenzer R., Ditmar P., Winsemius H.C., Savenije H.H.G., 2008, A comparison of global and regional GRACE models for land hydrology, Survey Geophysical, 29, pp 335-359.

Liu X., 2008, Global Gravity Field Recovery from satellite-to-satellite tracking data with the acceleration approach, $\mathrm{PhD}$ thesis published in Publications on Geodesy, 68.

Swenson S., 2011, Restoring Signal Loss in GRACE Terrestrial Water Storage Estimates, Hydrology and Earth System Sciences Discussions, 8/1827/2011.

Tapley B.D., Reiger Ch., 2008, The GRACE Mission, Status and Results, 2nd International GOCE User Workshop, Italy.

Veselinova-Renglova E., 2007, A Dynamic Geoid Model for Canada, A thesis submitted to the faculty of graduate studies in partial fulfillment of the requirements for the degree of doctor of philosophy, UCGE Reports Number 20261.

Zhang Z.Z., Chao B.F., Lu Y., Hsu H.T., 2009, An effective filtering for GRACE time-variable gravity: Fan filter, Geophysical Research Letters, Vol. 36, DOI: 10.1029/2009GL039459

http://www.eea.europa.eu/data-and-maps/data/biogeographical-regions-europe-2008/zippedshapefile-format-vector-polygon 